\title{
Drag and Reynolds stress distribution within submerged vegetation canopies
}

\author{
Nina Nikora $^{1,1}$, and Vladimir Nikora ${ }^{2}$ \\ ${ }^{1}$ School of Engineering, University of Aberdeen, Aberdeen, United Kingdom \\ ${ }^{2}$ School of Engineering, University of Aberdeen, Aberdeen, United Kingdom
}

\begin{abstract}
In the last few decades extensive studies focusing on both qualitative and quantitative descriptions of the Reynolds stress within aquatic canopies have been carried out. Although these studies have advanced our knowledge of mechanics of flow-vegetation interactions, further research in this area is still required. In particular, there is a need for development of new simple physically-based relationships describing the Reynolds stress profiles within submerged vegetation canopies. This paper addresses this issue and proposes a physically justified formulation for the Reynolds stress profile within the canopy region.
\end{abstract}

\section{Introduction}

Many phenomena in open-channel flows such as flow resistance, transport of pollutants, deposition and erosion of sediments are directly influenced by the primary Reynolds stress. Although for flows over smooth and sedimentary rough beds extensive data on Reynolds stress are already available, for vegetated flows such information remains limited, especially for the region within vegetation canopies. The available data suggest that the Reynolds stress in vegetated flows peaks around the top of the canopy and then it rapidly decays downward (e.g., Nepf and Vivoni, 2000; Poggi et al., 2004). The sharp decrease in the Reynolds stress within the canopy region is mainly caused by the drag due to vegetation elements. To predict vertical profiles of the Reynolds stress for flows with submerged vegetation researchers proposed a range of models (e.g., Lopez and Garcia, 2001). Although the past studies have provided some insights into the Reynolds stress distribution for aquatic flows, further research is still required. In particular, development of simple physically-based relationships for the Reynolds stress profiles within the canopy region is needed, as it is important for solving a range of practical problems, e.g., characterization of hydraulic habitat, sedimentation and passive substance transport within canopies. The goal of this paper, therefore, is to develop a physically justified relationship of the Reynolds stress distribution within a submerged vegetation canopy. In the following section, a relationship describing the vertical profile of the Reynolds stress within the canopy is derived first. This is followed by a description of experimental data and methods. Then, the proposed formulation is tested using extensive laboratory experiments and the effects of flow and vegetation characteristics on the driving parameters are identified.

\footnotetext{
1 Corresponding author: n.nikora@,abdn.ac.uk
} 


\section{Relationship for the Reynolds stress distribution within the vegetation canopy}

In general, the vertical distribution of the Reynolds stress in open-channel flows with vegetated beds can be complex, especially in the near bed region where the time-averaged flow is spatially heterogeneous. Hence, the use of the time-(ensemble-) averaged hydrodynamic equations for this region is not practicable due to high spatial variability of flow velocities and turbulence characteristics. To resolve this issue, researchers use the double-averaged (in both time and space domains) Navier-Stokes (DANS) equations that explicitly contain important additional terms such as form-induced stresses and viscous and form drag terms for the flow region within the canopy (e.g., Nikora et al., 2007). Considering steady, uniform 2D open-channel flow within the vegetation canopy and neglecting effects of vegetation porosity the DANS momentum equation for the longitudinal velocity component can be presented as:

$$
0=g S_{e}+\frac{1}{\rho} \frac{d \tau}{d z}-F_{D x}
$$

where $S_{e}$ is the energy slope, which for $2 \mathrm{D}$ uniform flow is equal to the bed slope $S_{b} ; z$ is the vertical coordinate (with origin at a channel bed); $F_{D x}=F_{V x}+F_{P x}, F_{V x}$ and $F_{P x}$ are the total drag, the viscous and form (pressure) drag forces per unit fluid mass, respectively; and $\tau$ is the total fluid stress (i.e., the total vertical momentum flux $\tau / \rho=-\left\langle\overline{u^{\prime} w^{\prime}}\right\rangle-\langle\tilde{u} \tilde{w}\rangle+\langle v \partial \bar{u} / \partial z\rangle$, where the first term on the right-hand side of $\tau / \rho$ is the spatially-averaged Reynolds stress, the second term is the form-induced (also known as dispersive) stress, and the third term is the double-averaged viscous stress). It is worth noting, that in high-Reynolds number openchannel flows with submerged high-density vegetation the main contributor to the total vertical momentum flux $\tau / \rho$ is $-\left\langle\overline{u^{\prime} w^{\prime}}\right\rangle$ (Poggi et al., 2004). Poggi et al. (2004) found that for the dense canopies contribution of $-\langle\tilde{u} \tilde{w}\rangle$ to $\tau / \rho$ is less than $5 \%$. Furthermore, above the viscous sub-layer $\langle v \partial \bar{u} / \partial z\rangle$ is negligible compared to $-\left\langle\overline{u^{\prime} w^{\prime}}\right\rangle$.

The total drag force $F_{D x}$ in (1) is commonly parameterized using a relationship:

$$
F_{D x}=0.5 C_{D} a\langle\bar{u}\rangle^{2}
$$

where $C_{D}$ is the drag coefficient; $a$ is vegetation density, i.e., the total frontal vegetation area per unit fluid volume; and $\langle\bar{u}\rangle$ is the local double-averaged velocity.

Equation (2) shows that for the homogeneous vegetation with height-independent density $a$ (i.e., $a=$ const), the drag force $F_{D x}$ may change with the vertical coordinate $z$ only if $C_{D}$ and/or $\langle\bar{u}\rangle$ change with $z$. Considering the findings of the experimental studies of vertical profiles of $C_{D}$ and $\langle\bar{u}\rangle$ (Dunn et al., 1996; Nepf and Vivoni, 2000), we can subdivide the vegetation canopy into two regions: the lower canopy (LC) and the upper canopy (UC) (Fig. 1). Within the LC region, the flow is mainly controlled by the balance of the gravity and total drag forces, while the total vertical momentum flux being negligible (Fig. 1). In this canopy region, the flow velocity and drag coefficient profiles are approximately constant. Thus, from Eq. (1) it follows that within the LC region the total drag force is approximately constant $F_{D x}=F_{L C}=g S_{b}$. In the UC, the total vertical momentum flux $\tau / \rho$ significantly contributes 
to the momentum balance, as schematically shown in Fig. 1. Within this region, the mean velocity increases with increase in $z$, resulting in decreasing $C_{D}$ following a relationship $C_{D} \sim\langle\bar{u}\rangle^{-\beta}$, where for submerged flexible vegetation $\beta \approx 2$ (Dunn et al., 1996; Nepf and Vivoni, 2000). Hence, within the UC region we have $C_{D}\langle\bar{u}\rangle^{2} \approx$ const and, therefore, the total drag force $F_{D x}=F_{U C}$ is also likely to be approximately constant, similar to the situation in the LC region. It is worth mentioning that an assumption $C_{D}\langle\bar{u}\rangle^{2} \approx$ const is supported by experimental data for the homogeneous flexible vegetation with height-independent density (Dunn et al., 1996; Nepf and Vivoni, 2000). However, further research is required to verify applicability of this assumption for the nonhomogeneous flexible vegetation, e.g., with branched morphology.

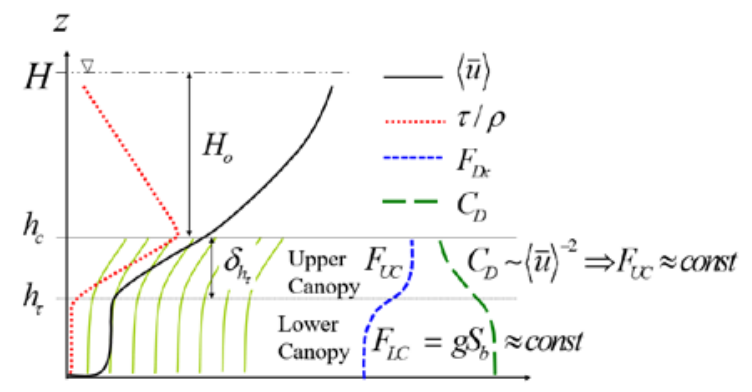

Fig. 1. Conceptual representation of the vertical profiles of the Reynolds stress, drag coefficient, and total drag force within the vegetation canopy.

Thus, assuming that the drag force within the canopy is approximately constant after integration of Eq. (1) we obtain a linear relationship for the total fluid stress within the UC region:

$$
\tau(z) / \rho=\left(F_{D x}-g S_{b}\right) z+C
$$

At $z=h_{\tau}$, where the total vertical momentum flux $\tau\left(h_{\tau}\right) / \rho=0$ (Fig. 1), the integration constant is equal to $C=-\left(F_{D x}-g S_{b}\right) h_{\tau}$. The elevation $z=h_{\tau}$ subdivides the vegetation canopy into the LC and UC regions with different momentum transport and drag-forming mechanisms. In the UC, the momentum transport is dominated by the total vertical momentum flux, while in the LC it is dominated by the gravity effect, as was first pointed out by Nepf and Vivoni (2000). Parameters $h_{\tau}$ and $\delta_{h_{\tau}}=h_{c}-h_{\tau}$ are the thicknesses of the UC and LC regions, respectively; they may be considered as integral measures of the flowvegetation interactions. Finally, substituting $C=-\left(F_{D x}-g S_{b}\right) h_{\tau}$ into (3) the relationship for the distribution of the total fluid stress within a submerged vegetation canopy can be summarized as:

$$
\tau(z) / \rho \begin{cases}\tau_{U C}(z) / \rho=\left(F_{D x}-g S_{b}\right)\left(z-h_{\tau}\right) & \text { for } z \geq h_{\tau} \\ \tau_{L C}(z) / \rho=0 & \text { for } z<h_{\tau}\end{cases}
$$

Considerations presented above allow us to obtain the ratio of the drag force $F_{U C}$ in the UC to the drag force $F_{L C}$ in the LC, i.e., $F_{U C} / F_{L C}$. Expressing each term of Eq. (1) individually for the LC and UC regions (Fig. 1) and integrating it one obtains:

$$
g S_{b} h_{c}+\tau_{h_{c}} / \rho-F_{U C}\left(h_{c}-h_{\tau}\right)-F_{L C} h_{\tau}=0
$$


where $\tau_{h_{c}}$ is the total fluid stress at the top of the canopy. Recalling that the drag force in the LC is $F_{L C}=g S_{b}$ (Fig. 1) and rearranging (5), we obtain:

$$
\frac{F_{U C}}{F_{L C}}=1+\frac{\tau_{h_{c}} / \rho}{g S_{b}\left(h_{c}-h_{\tau}\right)}
$$

The ratio of drag forces (6) is a measure of the relative contributions of the UC and LC regions to the total momentum sink (drag) within the entire vegetation canopy. This ratio is likely to be controlled by the flow and vegetation characteristics. For steady and uniform 2D flow $\tau_{h_{c}} / \rho=g S_{b} H_{o}$ ( $H_{o}$ is the depth of the overflow above the vegetation canopy). Substituting it into Eq. (6) we derive:

$$
\frac{F_{U C}}{F_{L C}}=\frac{H}{h_{c}-h_{\tau}}-\frac{h_{\tau}}{h_{c}-h_{\tau}}=\left(1-\frac{h_{\tau}}{h_{c}}\right)^{-1} \frac{H}{h_{c}}-\left(\frac{h_{c}}{h_{\tau}}-1\right)^{-1}
$$

Equation (7) shows that the dependence of the ratio of drag forces on the relative submergence is in general non-linear. For emergent conditions, i.e., $H / h_{c}=1$ and $h_{\tau} / h_{c}=1$, the ratio $F_{U C} / F_{L C}=0$, implying that there is no UC region. When $H / h_{c} \rightarrow \infty$, e.g., for terrestrial canopies, the LC region becomes negligible. Previous studies have shown that for flexible vegetation $h_{\tau} / h_{c}$ reaches a constant value at $H / h_{c} \approx 2$ (Nepf and Vivoni, 2000) while for rigid vegetation it becomes constant at $H / h_{c} \approx 4$ (Nezu and Sanjou, 2008). Thus, for a relatively high submergence (e.g., $H / h_{c} \geq 2-4$ ) the ratio $h_{\tau} / h_{c}$ is approximately constant and Eq. (7) becomes linear, i.e.:

$$
F_{U C} / F_{L C}=b H / h_{c}+c
$$

where $b=\left(1-\left(h_{\tau} / h_{c}\right)_{\text {const }}\right)^{-1}$ and $c=-\left(1 /\left(h_{\tau} / h_{c}\right)_{\text {const }}-1\right)^{-1}$ are constants, as $\left(h_{\tau} / h_{c}\right)_{\text {const }} \approx$ const. Further research is needed to develop predictive methods to assess the key parameters $h_{\tau}$, or $b$ and $c$ in (8) for potential use in applications. These parameters are likely to be dependent on vegetation properties, e.g., stem/plant morphology.

Thus, using the DANS momentum equation for steady and uniform 2D open-channel flow within the vegetation canopy, a simple relationship (4) for the total fluid stress and an expression for the drag force ratio (7) are deduced. To test proposed relationships, an extensive programme of laboratory experiments was carried out. The details on the experimental data and their analysis are presented in the following section.

\section{Experiments and methods}

\subsection{Experimental set-up}

Laboratory experiments were carried out in a $12.5 \mathrm{~m}$ long and $B=0.3 \mathrm{~m}$ wide rectangular glass-sided tilting flume (Fig. 2). An adjustable weir located at the discharge tank was used to minimize backwater effects and extend the section of (quasi-)uniform flow. The water discharge $Q$ was measured by an electromagnetic flow meter Sitrans MAG $5100 \mathrm{~W}$. Ten piezometric intakes tapped along the centre line of the flume bed were used to measure water surface slope. The water depth $H$ and deflected canopy height $h_{c}$ were measured at ten 
evenly-spaced cross-sections along the flume using decimal rulers glued to the glass side wall of the flume.

A three-component Nortek down-looking ADV was used to measure instantaneous velocities Fig. 2. A sampling duration of $120 \mathrm{~s}$, sampling frequency of $25 \mathrm{~Hz}$, and standard ADV measurement volume of $0.25 \mathrm{~cm}^{3}$ were used. To conduct laboratory experiments, two types of artificial flexible garden grasses, i.e., EasyPlants (EP) and EverGreens (EG), have been selected (Fig. 2 and Table 1). The stems are arranged as groups of uniformly distributed plants weaved to a thin black plastic base (Fig. 2c). Each plant consisted of exactly 16 for EP100/EP50 and on average 20.8 (varied from 19 to 24) for EG100 individual stems of undeflected height 3.59 and $4.38 \mathrm{~cm}$, respectively. The key parameters for both grasses are shown in Table 1 , including porosity $\phi$ and the stem flexural rigidity $J$.

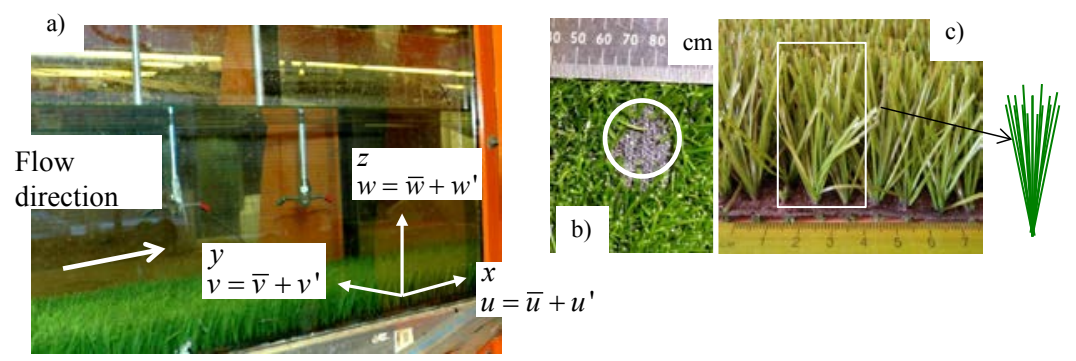

Fig. 2. Experimental set-up: a) Armfield flume fully covered by artificial flexible grass EP100; b) a measurement hole within the vegetation canopy EP100; c) side view of EP100.

The experiments involved measurements of the: (a) bulk hydraulic parameters (water depth, water surface level, flow rate, and channel bed slope); (b) vegetation characteristics (canopy height, density, geometrical and biomechanical characteristics, and porosity), and (c) instantaneous velocities measured at a single location in the middle of the flume (along and across the channel). The ranges of the main parameters are shown in Table 1.

Table 1. Grass characteristics and ranges of experimental parameters.

\begin{tabular}{|c|c|c|c|c|c|c|c|c|}
\hline $\begin{array}{l}\text { Grass } \\
\text { ID }\end{array}$ & $\begin{array}{c}a \\
(1 / \mathrm{m})\end{array}$ & $\begin{array}{l}\phi \\
(-) \\
\end{array}$ & $\begin{array}{c}J \\
\left(\mathrm{Nm}^{2}\right) \\
\end{array}$ & $\begin{array}{l}\text { Exp. } \\
\text { No }\end{array}$ & $\begin{array}{l}S_{b} \\
(\%) \\
\end{array}$ & $\begin{array}{c}U_{m}=\frac{Q}{B H} \\
(\mathrm{~m} / \mathrm{s})\end{array}$ & $\begin{array}{c}H / h_{c} \\
(-) \\
\end{array}$ & $\begin{array}{r}B / H \\
(-) \\
\end{array}$ \\
\hline \multirow{2}{*}{ EG100 } & \multirow{2}{*}{372} & \multirow{2}{*}{0.98} & \multirow{2}{*}{$42.29 \mathrm{E}-07$} & \multirow{2}{*}{32} & 0.05 & 0.15 & 3.00 & 0.71 \\
\hline & & & & & 1.00 & 0.85 & 9.72 & 2.30 \\
\hline \multirow{2}{*}{ EP100 } & \multirow{2}{*}{268} & \multirow{2}{*}{0.97} & \multirow{2}{*}{7.73 E-07 } & \multirow{2}{*}{31} & 0.05 & 0.17 & 3.49 & 0.75 \\
\hline & & & & & 0.40 & 0.57 & 11.81 & 2.52 \\
\hline \multirow{2}{*}{ EP50 } & \multirow{2}{*}{134} & \multirow{2}{*}{0.99} & \multirow{2}{*}{7.73 E-07 } & \multirow{2}{*}{29} & 0.05 & 0.17 & 3.64 & 0.74 \\
\hline & & & & & 0.40 & 0.56 & 12.66 & 2.38 \\
\hline
\end{tabular}

\subsection{Data analysis}

In the proposed relationship (4), the total fluid stress is approximately equal to the Reynolds stress, i.e., $\tau(z) / \rho \approx-\left\langle\overline{u^{\prime} w^{\prime}}\right\rangle(z)$, since contributions from both form-induced (Poggi et al., 2004) and viscous stresses are negligible. Therefore (4) can serve as a relationship of the Reynolds stress distribution within the canopy. In the present study, the primary Reynolds stress is determined as:

$$
R S_{v e r}=\left({\overline{u^{\prime} w^{\prime}}}^{2}(z)+{\overline{v^{\prime} w^{\prime}}}^{2}(z)\right)^{1 / 2}
$$


where the second term on the right-hand side of (9) is used to eliminate potential probe misalignment effects in the computation of the primary Reynolds stress.

The parameters of Eq. (4) for the UC region, i.e., the gradient $A=\left(F_{U C}-g S_{b}\right)$ and intercept $C=-\left(F_{U C}-g S_{b}\right) h_{\tau}=-A h_{\tau}$, are found by best fitting the measured profiles (9) to Eq. (4) using the experimental data within the range $z / h_{c}=0.55-0.9$. This range has been determined by visual assessment of the measured vertical profiles of the Reynolds stress. Then, using the obtained parameters $A$ and $C$, the constant drag force $F_{U C}$ for the UC and the level $h_{\tau}$ of the negligible vertical turbulent transport of momentum are determined as:

$$
\begin{gathered}
F_{U C}=A+g S_{b} \\
h_{\tau}=-C /\left(F_{U C}-g S_{b}\right)=-C / A
\end{gathered}
$$

To compare estimates of $h_{\tau}$ from (11) with the penetration depth $h_{p}$ proposed in Nepf and Vivoni (2000), their "10\%-technique" was applied to the vertical profiles of Reynolds stress (9). Specifically, the penetration depth is estimated as the distance from the channel bed to an elevation where the Reynolds stress decays to $10 \%$ of its maximum value.

\section{Tests of the Reynolds stress relationship}

The Reynolds stress relationship (4) is tested using the experimental data for all three data sets described in section 3.1. The data demonstrate that Eq. (4) approximates the Reynolds stress distribution within the upper canopy very well. This implies that the parameters $A$ and $C$ in (10) and (11) can be used to estimate the upper canopy drag force $F_{U C}$ and the depth of negligible vertical turbulent transport of momentum $h_{\tau}$.

The depth of negligible vertical turbulent transport of momentum $h_{\tau}$ is compared in Fig. 3 to the penetration depth estimated using Nepf and Vivoni's (2000) 10\%-technique. As expected, the data show that $h_{\tau} / h_{c}$ values are approximately $10 \%$ smaller than $h_{p} / h_{c}$.

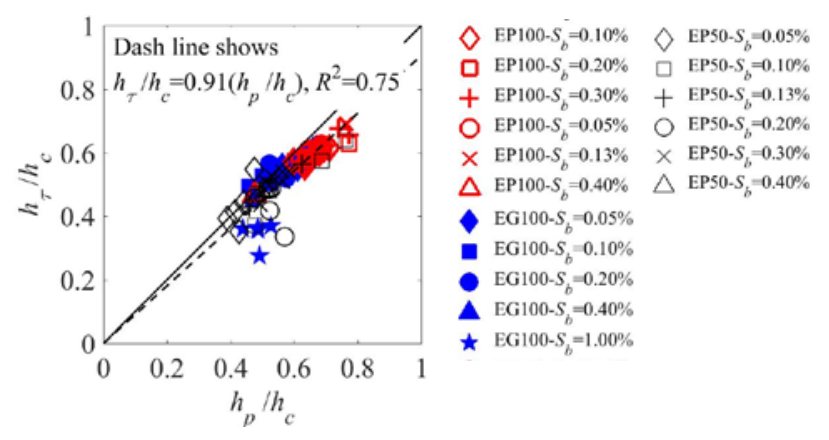

Fig. 3. Correlation of $h_{\tau} / h_{c}$ with the penetration depth $h_{p} / h_{c}$ estimated using Nepf and Vivoni's (2000) 10\%-technique. Solid line denotes $y=x$ relationship.

Fig. 4a demonstrates the dependence of $F_{U C} / F_{L C}$ on relative flow submergence $H / h_{c}$. The data show that with increase in $H / h_{c}$ the ratio of drag forces $F_{U C} / F_{L C}$ increases. For an experimental range of $H / h_{c}$, covered in the present study, i.e., $H / h_{c}=3-12.7$, the 
following ranges of $F_{U C} / F_{L C}$ have been obtained: 3.1-13.6 for EG100, 3.1-12.2 for EP100 and 3.7-11.8 for EP50. Fig. 4a shows that for a given value of relative submergence, the ratio of drag forces $F_{U C} / F_{L C}$ increases with increase in vegetation density. In general, the drag force ratio $F_{U C} / F_{L C}$ may be comparable to the ratio of the turbulent stress gradient $\partial\left\langle\overline{-u^{\prime} w^{\prime}}\right\rangle / \partial z$ to the pressure gradient $g \partial H / \partial x$ (or to the gradient of the channel bed $\partial z_{b} / \partial x$ for uniform flow, where $z_{b}$ is the bed elevation), proposed in Nepf and Vivoni (2000). Indeed, taking into account that $g \partial z_{b} / \partial x=g S_{b}=F_{L C}$ and considering the flow within the UC region, Eq. (1) can be re-written as:

$$
\frac{F_{U C}}{F_{L C}}-1=\frac{1}{\rho} \frac{d \tau / d z}{F_{L C}}
$$

The right-hand term in (12) is equivalent to the ratio $\left(\partial\left\langle\overline{u^{\prime} w^{\prime}}\right\rangle / \partial z\right) /\left(g \partial z_{b} / \partial x\right)$. In agreement with (12), the Nepf and Vivoni (2000) data in Fig. 4a, which covers flexible lowdensity vegetation with $a h_{c}=0.88$, are consistent with the drag force ratio $F_{U C} / F_{L C}$ values.
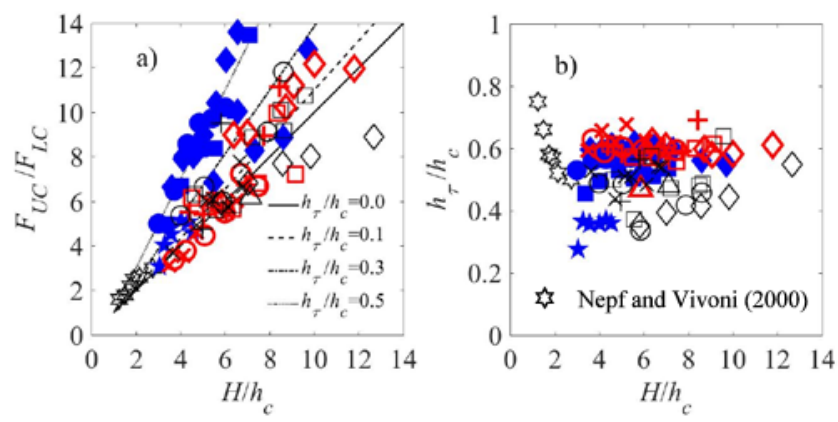

Fig. 4. Dependence of $F_{U C} / F_{L C}$ (a) and $h_{\tau} / h_{c}$ (b) on $H / h_{c}$. Black lines of different styles in plot (a) show tests of Eq. (8) for a range of $h_{\tau} / h_{c}$ values. Symbols as in Fig. 3.

Fig. 4a also serves as a test of Eq. (7), covering a range of $h_{\tau} / h_{c}$ from 0 (the turbulent stress penetrates to the bed) to 0.5 (the total vertical momentum flux is fully absorbed within the upper half of the canopy). The experimental data demonstrate that Eq. (7) approximates the drag force ratio fairly well. For example, the EG100 data points closely collapse around a straight theoretical line for $h_{\tau} / h_{c}=0.5$, consistent with Eq. (7) and the average value of the unconfined limit of $h_{\tau} / h_{c}=0.53$ (Fig. 4b). A good agreement between the experimental data and Eq. (7) suggests that when the penetration depth reaches its unconfined limit, i.e., becomes independent of relative submergence and only depends on vegetation parameters, the dependence of $F_{U C} / F_{L C}$ on $H / h_{c}$ can be described by a simple linear relationship (8).

The dependence of $h_{\tau} / h_{c}$ on the relative submergence is illustrated in Fig. 4b. The data show that for a range of $H / h_{c}$, investigated in the present study, the ratio $h_{\tau} / h_{c}$ remains approximately constant with increase in the relative submergence, consistent with expectations. Fig. $4 \mathrm{~b}$ also shows that for a given $H / h_{c}$ value, the ratio $h_{\tau} / h_{c}$ increases with increase in the vegetation density. Furthermore, for given values of both the vegetation 
density and relative submergence, increasing bed slope leads to decrease in $h_{\tau} / h_{c}$, as has already been seen in Fig. 3.

Unfortunately, the present study does not cover $H / h_{c}<3$. However, based on the results of Nepf and Vivoni (2000) who demonstrated that the relative penetration depth decreased from around 1 for emergent vegetation, i.e., $H / h_{c}=1$, to an unconfined limit at $H / h_{c} \approx 2$ (their data are also shown in Fig. 4b), it is reasonable to assume that $h_{\tau} / h_{c}$ should be decreasing within the range $H / h_{c}=1-3$. This assumption may be further supported by the results of Nezu and Sanjou (2008) who also found that the relative penetration depth reached its unconfined limit, but at much higher value of the flow submergence $H / h_{c} \approx 4$. The noted discrepancy between Nezu and Sanjou (2008) and Nepf and Vivoni (2000) data most likely relates to the significant differences in plant rigidities (flexible in Nepf and Vivoni (2000) versus rigid in Nezu and Sanjou (2008)), morphologies, and densities. Potential effects of secondary currents should also not be dismissed.

\section{CONCLUSIONS}

A new physically-based relationship describing the Reynolds stress profiles within the submerged vegetation canopy is proposed. The relationship is deduced using the DANS momentum equation for steady and uniform 2D open-channel flow within the vegetation canopy. The key parameter of the proposed relationship $h_{\tau}$ subdivides the canopy into the upper and lower canopy regions with different momentum transport and drag-forming mechanisms. Within the upper canopy the momentum transport is dominated by the total vertical momentum flux, while in the lower canopy part it is dominated by the gravity action. The ratio of the drag forces acting in the upper and lower canopy regions is approximated by a simple non-linear relationship that links this ratio to the relative penetration depth $h_{\tau} / / h_{c}$ and flow submergence $H / h_{c}$. When $h_{\tau} / h_{c}$ approaches a constant value above some threshold flow submergence the relationship $F_{U C} / F_{L C}=f\left(H / h_{c}\right)$ becomes linear. The drag force ratio $F_{U C} / F_{L C}$ may be interpreted as a measure of the relative contributions of the upper and lower canopy regions to the total momentum sink (drag) occurring within the entire vegetation layer. The experimental data support the proposed relationship fairly well, consistent with the assumption that the total drag within the submerged vegetation canopy is approximately constant within both the upper and lower canopy regions.

\section{REFERENCES}

1. Dunn, C., Lopez, F. and Garcia, M. Hydr. Eng. Series No. 51 (1996)

2. Lopez, F. and Garcia, M.H. Hydr. Eng. J. 127(5) (2001)

3. Nepf, H.M. and Vivoni, E.R. Geoph. Res. J. 105(C12) (2000)

4. Nezu, I. and Sanjou, M. Hydro-envir. Res. J. 2 (2008)

5. Nikora, V., McEwan, I., McLean, S., Coleman, S., Pokrajac, D. and Walters, R. Hydr. Eng. J. ASCE, 133(8) (2007)

6. Poggi, D., Katul, G.G and Albertson, J. D. Bound.-Lay. Meteor. 111 (2004) 